\title{
Cosmology Under Milne's Shadow
}

\author{
Michat J. Chodorowski ${ }^{\mathrm{A}}$ \\ A Copernicus Astronomical Center, 00-716 Warsaw, Poland. Email: michal@camk.edu.pl
}

Received 2005 April 28, accepted 2005 July 27

\begin{abstract}
Based on the magnitude-redshift diagram for the sample of supernovae Ia analyzed by Perlmutter et al. (1999), Davis \& Lineweaver (2004) ruled out the special relativistic interpretation of cosmological redshifts at a confidence level of $23 \sigma$. Here, we critically reassess this result. Special relativity is known to describe the dynamics of an empty universe, by means of the Milne kinematic model. Applying only special relativistic concepts, we derive the angular diameter distance and the luminosity distance in the Milne model. In particular, in this model we do not use the underlying metric in its Robertson-Walker form, so our exposition is useful for readers without any knowledge of general relativity. We do however, explicitly use the special relativistic Doppler formula for redshift. We apply the derived luminosity distance to the magnitude-redshift diagram for supernovae Ia of Perlmutter et al. (1999) and show that special relativity fits the data much better than that claimed by Davis \& Lineweaver. Specifically, using these data alone, the Milne model is ruled out only at a $2 \sigma$ level. Alhough not a viable cosmological model, in the context of current research on supernovae Ia it remains a useful reference model when comparing predictions of various cosmological models.
\end{abstract}

Keywords: methods: analytical — galaxies: distances and redshifts — cosmology: observations cosmology: theory

\section{Introduction}

In a recent paper, Davis \& Lineweaver (2004) attempted to clarify several common misconceptions about the expansion of the universe. In particular, they convincingly pointed out that uniform expansion of an infinite universe implies that very distant galaxies recede from us with superluminal recession velocities. Moreover, we can observe such galaxies. This does not violate special relativity (SR), because their velocities are not measured in any observer's inertial frame. They are measured in the so-called reference frame of Fundamental Observers, for which the universe looks homogeneous and isotropic.

Unfortunately while Davis \& Lineweaver not only clarified some misconceptions, they also created a new misunderstanding. They claimed to 'observationally rule out the SR Doppler interpretation of cosmological redshifts at a confidence level of $23 \sigma^{\prime}$. (The special relativistic interpretation of redshift is kinematic, i.e. the Doppler effect.) As we will explain later, Davis \& Lineweaver did not apply SR properly. Specifically, they did not consistently use the definition of the luminosity distance, $D_{\mathrm{L}}$. SR is known to describe the dynamics of an empty universe (Peacock 1999; Longair 2003). Their error led Davis \& Lineweaver to an expression for the luminosity distance as a function of redshift that was entirely different from that for an empty universe.

In the framework of general relativity, the calculation of $D_{\mathrm{L}}$ for an empty universe is straightforward. However, here we will present also an alternative approach, based entirely on SR. Namely, we will derive $D_{\mathrm{L}}$ applying the kinematic cosmological model of Milne (1933). This approach is useful for readers without any knowledge of general relativity. It elucidates the meaning of time and kinematics in cosmology. The Milne model offers also an interesting insight into all Friedman-Robertson-Walker (FRW) cosmological models.

The paper is organized as follows. We begin in Section 2 by deriving the angular diameter distance for an empty universe using the FRW framework. Next, we present the corresponding derivation in the Milne model. In Section 3 we derive the luminosity distance in the Milne model and compare it to the angular diameter distance. In Section 4 we present the resulting magnitude-redshift diagram for supernovae Ia, and present a summary in Section 5.

\section{Angular Diameter Distance}

For reference, let us first recall the derivation of the angular diameter distance for an empty universe in the FRW framework. The metric of a homogeneous and isotropic universe is given by the Robertson-Walker line element:

$$
c^{2} \mathrm{~d} s^{2}=c^{2} \mathrm{~d} t^{2}-a^{2}(t)\left[\mathrm{d} x^{2}+R_{\mathrm{o}}^{2} S^{2}\left(x / R_{\mathrm{o}}\right) \mathrm{d} \Omega\right] .
$$

Here,

$$
\mathrm{d} \Omega=\mathrm{d} \theta^{2}+\sin ^{2} \theta \mathrm{d} \phi^{2},
$$

and $R_{\mathrm{O}}^{-2}$ is the (present) curvature of the universe. The function $S(x)$ equals $\sin (x), x$, and $\sinh (x)$ for a closed, flat, and open universe, respectively. The function $a(t)$ is called a scale factor and relates the physical, or proper, coordinates of a galaxy, $\mathbf{r}$, to its fixed or comoving coordinates, $\mathbf{x}: \mathbf{r}=a \mathbf{x}$. This function accounts for the expansion of the universe; its detailed time dependence is determined by 
the Friedman equations. We normalize $a$ so that at the present time, $a\left(t_{\mathrm{o}}\right)=1$. For an open universe, the Friedman equations yield

$$
R_{\mathrm{o}}=\frac{c H_{\mathrm{o}}^{-1}}{\left(1-\Omega_{\mathrm{o}}\right)^{1 / 2}},
$$

where $H_{\mathrm{o}}$ is the present value of the Hubble constant and $\Omega_{\mathrm{O}}$ is the present value of the total (energy) density in the universe, in units of the so-called critical (energy) density.

Let us work out the angular size of an object of proper length $\Delta y$, perpendicular to the radial coordinate at redshift $z$. The relevant spatial component of the metric (Eqns $1 \& 2$ ) is the term in $\mathrm{d} \theta$. The proper length $\Delta y$ of an object at redshift $z$, corresponding to scale factor $a\left(t_{\mathrm{e}}\right)$, for an open universe is

$$
\Delta y=a\left(t_{\mathrm{e}}\right) R_{\mathrm{o}} \sinh \left(x / R_{\mathrm{o}}\right) \Delta \theta=D_{\mathrm{A}} \Delta \theta,
$$

where we have introduced the angular diameter distance $D_{\mathrm{A}}=a\left(t_{\mathrm{e}}\right) R_{\mathrm{O}} \sinh \left(x / R_{\mathrm{O}}\right)$. Here, $t_{\mathrm{e}}$ is the time of emission of photons. Since $a\left(t_{\mathrm{e}}\right)^{-1}=1+z$, we have

$$
D_{\mathrm{A}}=R_{\mathrm{o}} \frac{\sinh \left(x / R_{\mathrm{o}}\right)}{1+z} .
$$

For an empty universe, $\Omega_{\mathrm{o}}=0$, hence $R_{\mathrm{o}}=c H_{\mathrm{o}}^{-1}$. Moreover, then $a(t)=H_{\mathrm{o}} t$ and the equations of null radial geodesics are easy to integrate. The result is $x=$ $c H_{\mathrm{o}}^{-1} \ln (1+z)$. Substituting this into Eqn (5) and using the definition of the hyperbolic sine, we obtain

$$
D_{\mathrm{A}}(z)=c H_{\mathrm{o}}^{-1} \frac{z(1+z / 2)}{(1+z)^{2}} .
$$

This is the angular diameter distance for an empty universe, derived in the FRW framework.

In the Milne model, the cosmic arena of physical events is the pre-existing Minkowski spacetime. In the origin of the coordinate system, $O$, at time $t=0$ an 'explosion' takes place, sending radially fundamental observers with constant velocities in the range of speeds $(0, c)$. The fundamental observer with velocity $v, F_{v}$, carries a rigid rod of length $\Delta y$, oriented perpendicularly to the line of sight of the observer at $O$. At time $t_{\mathrm{e}}$ this rod emits photons. At the photons' arrival time at $O, t_{\mathrm{o}}$, the rod subtends at $O$ an angle

$$
\Delta \phi=\Delta y / r_{\mathrm{e}},
$$

where $r_{\mathrm{e}}$ is the distance from $O$ to $F_{v}$ at the time of emission of the photons, $t_{\mathrm{e}}$. We have $t_{\mathrm{o}}=t_{\mathrm{e}}+t_{\mathrm{t}}$, where $t_{\mathrm{t}}$ is the travel time of the photons. Since

$$
t_{\mathrm{e}}=\frac{r_{\mathrm{e}}}{v}
$$

and

$$
t_{\mathrm{t}}=\frac{r_{\mathrm{e}}}{c},
$$

we obtain

$$
r_{\mathrm{e}}=c t_{\mathrm{o}} \frac{\beta}{1+\beta},
$$

where $\beta=v / c$. The special relativistic formula for the Doppler effect is

$$
1+z=\left(\frac{1+\beta}{1-\beta}\right)^{1 / 2},
$$

where $z$ is the photons' redshift; hence,

$$
\beta=\frac{(1+z)^{2}-1}{(1+z)^{2}+1} .
$$

Using Eqn (12) in Eqn (10) yields

$$
r_{\mathrm{e}}=c t_{\mathrm{O}} \frac{z(1+z / 2)}{(1+z)^{2}} \text {. }
$$

Since in the Milne model, for any time $t$ and for any fundamental observer $F_{v}, r=v t$, the observer at $O$ observes the Hubble flow: $v=H r$, where the Hubble constant is $H=t^{-1}$. Hence, $t_{\mathrm{o}}=H_{\mathrm{o}}^{-1}$. The angular diameter distance is defined via the equation $\Delta y=D_{\mathrm{A}} \Delta \phi$. Therefore, using Eqn (7) we obtain finally

$$
D_{\mathrm{A}}(z)=r_{\mathrm{e}}=c H_{\mathrm{o}}^{-1} \frac{z(1+z / 2)}{(1+z)^{2}} .
$$

In the above derivation we have applied special relativity a number of times. First, writing Eqn (9) we have followed its central assumption, that the velocity of light is always $c$, regardless the relative motion of the emitter and the observer. Secondly, we have applied the special relativistic, kinematic interpretation of redshift - the Doppler effect — and used Eqn (11) for it. Finally, writing Eqn (7) we have assumed that the geometry of space is Euclidean.

Eqn (14) exactly coincides with Eqn (6) for $D_{\mathrm{A}}$ for an empty universe, the latter derived from the metric in its FRW form. Our second derivation, which was purely special relativistic, employed other (i.e., conventional Minkowskian) definitions of distance and time. Still, we arrived at the same formula for $D_{\mathrm{A}}$. This is so because this formula relates direct observables: proper (i.e., rest frame) size of an object to its angular size and redshift. Regardless of what are the definitions of coordinates in a given coordinate system, their consistent application should lead to the same result in terms of observables!

\section{Luminosity Distance}

The relation between the luminosity distance, $D_{\mathrm{L}}$, and the angular diameter distance is

$$
D_{\mathrm{L}}=(1+z)^{2} D_{\mathrm{A}} \text {. }
$$

However, this is true in general relativity; let's check whether this holds also in the Milne model. Let's place a source of radiation at the origin of the coordinate system, $O$. We assume again that at time $t=0$ at $O$ an 'explosion' takes place, sending radially fundamental observers 
with constant velocities in the range of $(0, c)$. At time $t_{\mathrm{e}}$ the source emits photons, which at time $t_{\mathrm{o}}$ reach a fundamental observer moving with velocity $v$, such that

$$
v t_{\mathrm{o}}=c\left(t_{\mathrm{o}}-t_{\mathrm{e}}\right) .
$$

If the source emits continuously photons with constant bolometric luminosity $L$, the observer at $r_{\mathrm{o}}=v t_{\mathrm{o}}$ receives a flux of radiation with bolometric intensity

$$
f=\frac{L}{4 \pi r_{\mathrm{o}}^{2}(1+z)^{2}} .
$$

The factor $(1+z)^{2}$ in the denominator is due to the Doppler effect. Specifically, one factor $1+z$ is due to the fact that the wavelength, and so the energy, of the observed photons is redshifted. The second factor $1+z$ is due to the fact that photons, emitted in the time interval $\Delta t_{\mathrm{e}}$, arrive to the observer in the time interval $t_{\mathrm{o}}=(1+z) \Delta t_{\mathrm{e}}$.

The luminosity distance is defined by the equation $f=L /\left(4 \pi D_{\mathrm{L}}^{2}\right)$, hence

$$
D_{\mathrm{L}}=(1+z) r_{\mathrm{o}}=(1+z) c \beta t_{\mathrm{o}} .
$$

Time $t_{\mathrm{o}}$ is the time of observation indicated by the clock at the source, but the fundamental observer is moving with respect to $O$, so according to SR, his clock delays compared to that at $O$. At the moment of observation, his clock shows time $\tau_{\mathrm{o}}=t_{\mathrm{o}} / \gamma$, where $\gamma=\left(1-\beta^{2}\right)^{-1 / 2}$. Therefore, we have

$$
D_{\mathrm{L}}=(1+z) c \tau_{\mathrm{o}} \beta \gamma
$$

Relative to the observer, however, this is the source that is moving. Using his clock, the observer deduces that since the Big Bang, the source has moved off to the distance $r_{\mathrm{o}}^{\prime}=v \tau_{\mathrm{o}}$. Because the source is moving, the distance $r_{\mathrm{o}}^{\prime}$ is length-contracted relative to its rest-frame value, $r_{\mathrm{o}}: r_{\mathrm{o}}^{\prime}=r_{\mathrm{o}} / \gamma$. Hence, the observer will agree that $r_{\mathrm{o}}=\gamma v \tau_{\mathrm{o}}=c \tau_{\mathrm{o}} \beta \gamma$, what yields Eqn (19).

Next, we have

$$
\beta \gamma=\frac{\beta}{(1-\beta)^{1 / 2}(1+\beta)^{1 / 2}}=(1+z) \frac{\beta}{1+\beta},
$$

where in the last equality we have used the SR formula for redshift, Eqn (11). Combined with Eqn (12), Eqns (19) \& (20) yield

$$
D_{\mathrm{L}}=(1+z)^{2} c \tau_{\mathrm{o}} \frac{z(1+z / 2)}{(1+z)^{2}}=c H_{\mathrm{o}}^{-1} z(1+z / 2) .
$$

Comparing with Eqn (14) we see that indeed $D_{\mathrm{L}}=$ $(1+z)^{2} D_{\mathrm{A}}$, in accordance with general relativity.

To derive the angular diameter distance, in Sec. 2 we have used the observer's rest-frame. To derive the luminosity distance, however, in the present section we have switched to the source's rest-frame. We have done so for simplicity of the resulting calculations. In particular, only in the latter frame is the radiation of the source isotropic, and one can apply simple Eqn (17) for the observed flux.
Deriving the two distances, we have placed either the observer or the source at a special position - at the center of expansion. Are then our results general? Yes: Although in the Milne model this center does indeed exist, every fundamental observer considers himself to be at the center of expansion! This can be easily seen in the non-relativistic regime. According to the Galilean transformation of velocities, the velocity of any observer $O^{\prime \prime}$ relative to another observer $O^{\prime}$ is $\mathbf{v}^{\prime}=\mathbf{v}-\mathbf{V}_{\text {rel }}$, where $\mathbf{v}$ and $\mathbf{V}_{\text {rel }}$ are respectively the velocity of $O^{\prime \prime}$ relative to $O$ and the velocity of $O^{\prime}$ relative to $O$. But by the construction of the model, the observer at $O$ observes the Hubble flow, so $\mathbf{v}=H_{\mathrm{o}} \mathbf{r}$ and $\mathbf{V}_{\text {rel }}=H_{\mathrm{o}} \mathbf{R}$, where $\mathbf{r}$ denotes the position of $O^{\prime \prime}$ relative to $O$ and $\mathbf{R}$ denotes the position of $O^{\prime}$ relative to $O$. Hence, $\mathbf{v}^{\prime}=H_{\mathrm{o}}(\mathbf{r}-\mathbf{R})=H_{\mathrm{o}} \mathbf{r}^{\prime}$, where $\mathbf{r}^{\prime}$ is the position of $O^{\prime \prime}$ relative to $O^{\prime}$. Thus, the observer $O^{\prime}$ observes an isotropic Hubble flow around him, so is located apparently at the center of expansion. The point is that this result holds also for relativistic velocities (Milne 1933; Rindler 1977). Strictly speaking, in the Hubble law the Hubble constant is an inverse of the local proper time of the observer at $O^{\prime}$. This is why in Eqn (21) we have identified $H_{\mathrm{o}}$ with $\tau_{\mathrm{o}}^{-1}$.

It is rather surprising why Milne, who insisted so much on an observables-oriented approach to cosmology, did not derive himself an explicit formula for the luminosity distance as a function of redshift. We will see, however, that he was close to it. The Appendix to his classical paper on kinematic relativity (Milne 1933) bears the title 'The apparent brightness of a receding nebula'. Its final formula describes 'the total light received by A (the observer) on his own photographic plate' in a fairly complex, integral form. This formula involves implicitly the luminosity distance, but in terms of the recession velocity of the nebula rather than its redshift. However, while the redshift of a distant nebula is a direct observable, its velocity is not. Extracting the luminosity distance from the formula and using the SR relation between velocity and redshift, Eqn (11), we rederive the formula for the luminosity distance given by our Eqn (21).

\section{Apparent Magnitude-Redshift Relation}

The apparent bolometric magnitude, $m_{\mathrm{B}}$, of a standard candle located at redshift $z$ is related to its absolute bolometric magnitude, $M$, by the equation

$$
m_{\mathrm{B}}=5 \log _{10} D_{\mathrm{L}}+25+M .
$$

Here, $D_{\mathrm{L}}$ is the luminosity distance, expressed in megaparsecs. Supernovae Ia turned out to be very good standard candles in the universe (see, for example, Perlmutter 2003). Perlmutter et al. (1997) cast the above equation to the form

$$
m_{\mathrm{B}}=5 \log _{10}\left(H_{\mathrm{o}} D_{\mathrm{L}}\right)+\mathcal{M}
$$

where

$$
\mathcal{M}=M-5 \log _{10} H_{\mathrm{o}}+25
$$




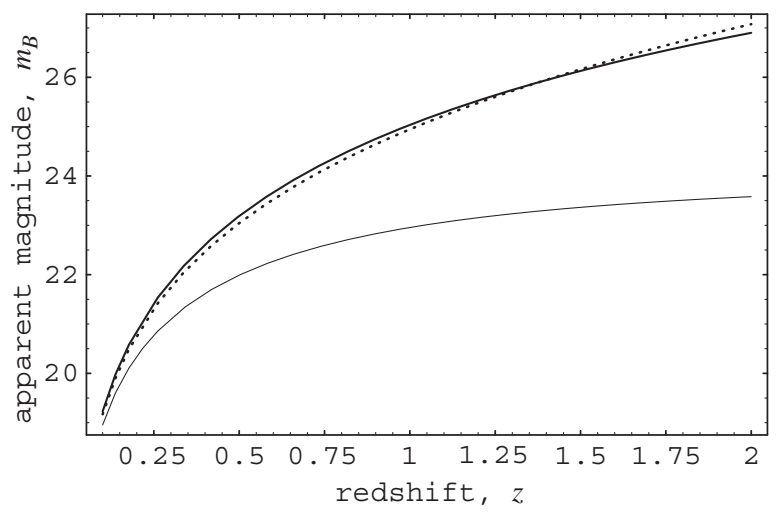

Figure 1 The magnitude-redshift diagram for supernovae Ia. The thick solid line shows the prediction of the currently favoured cosmological model, $\Omega_{\mathrm{m}}=0.28$ and $\Omega_{\Lambda}=0.72$. The thin solid line shows the prediction of special relativity according to Davis \& Lineweaver. The dotted line shows the correct prediction of special relativity.

is the magnitude zero-point, and the Hubble constant is expressed in $\mathrm{km} \mathrm{s}^{-1} \mathrm{Mpc}^{-1}$ (see eqns 1 \& 2 in Perlmutter et al. 1997). We prefer to rewrite Eqn (23) to the form

$$
m_{\mathrm{B}}=5 \log _{10}\left(\frac{D_{\mathrm{L}}}{c H_{\mathrm{O}}^{-1}}\right)+\widetilde{\mathcal{M}},
$$

where

$$
\widetilde{\mathcal{M}}=\mathcal{M}+5 \log _{10} c=\mathcal{M}+25+5 \log _{10} 2.998
$$

the argument of logarithm in Eqn (25) is explicitly dimensionless. Introducing Eqn (21) into Eqn (25) yields

$$
m_{\mathrm{B}}=5 \log _{10}[z(1+z / 2)]+\widetilde{\mathcal{M}} .
$$

This is the magnitude-redshift relation in the Milne model. It coincides exactly with the corresponding relation for an empty universe. In Figure 1 it is shown as a dotted line. For the magnitude zero-point we adopt the value $\mathcal{M}=-3.32$, calculated in Perlutter et al. (1997) and used also in Perlmutter et al. (1999).

To derive the luminosity distance $D_{\mathrm{L}}=(1+z) D$, Davis \& Lineweaver used the Hubble law:

$$
D=c H^{-1} \beta .
$$

On comparison with Eqn (18) we see that this leads to the correct expression for $D_{\mathrm{L}}$ provided we identify $H$ with $H_{\mathrm{o}}^{(\mathrm{s})}$, i.e. the value of the Hubble constant at the source and at the time of observation of photons. However, they identified $H$ with $H_{\mathrm{e}}^{(\mathrm{s})}$, i.e. the Hubble constant at the source but at the time of emission of photons:

$$
D^{(\mathrm{DL})}=c\left(H_{\mathrm{e}}^{(\mathrm{s})}\right)^{-1} \beta=c \beta t_{\mathrm{e}} .
$$

Combined with the SR expression for $\beta$ as a function of redshift, Eqn (12), the above equation yields

$$
D^{(\mathrm{DL})}=\frac{c}{H_{\mathrm{e}}^{(\mathrm{s})}} \frac{(1+z)^{2}-1}{(1+z)^{2}+1}
$$

(eqn 10 of Davis \& Lineweaver 2004). Next, Davis \& Lineweaver applied the equality

$$
H_{\mathrm{e}}^{(\mathrm{s})}=(1+z) H_{\mathrm{o}}^{(\mathrm{o})},
$$

correct in case of an empty universe. Let's check whether this holds also in the Milne model. First, $H_{\mathrm{e}}^{(\mathrm{s})}=t_{\mathrm{e}}^{-1}$ and $H_{\mathrm{o}}^{(\mathrm{o})}=\tau_{\mathrm{o}}^{-1}$, where $t$ and $\tau$ are the time measured (by Fundamental Observers) respectively at the source and at the observing point. Hence,

$$
\frac{H_{\mathrm{e}}^{(\mathrm{s})}}{H_{\mathrm{o}}^{(\mathrm{o})}}=\frac{\tau_{\mathrm{o}}}{t_{\mathrm{e}}}=\gamma^{-1} p^{-1}
$$

where $p \equiv t_{\mathrm{e}} / t_{\mathrm{o}}$. From Eqn (16) we have

$$
\beta=1-p
$$

so

$$
\gamma^{-1}=\left(1-\beta^{2}\right)^{1 / 2}=[p(2-p)]^{1 / 2} .
$$

Introducing the above into Eqn (32) yields

$$
\frac{H_{\mathrm{e}}^{(\mathrm{s})}}{H_{\mathrm{o}}^{(\mathrm{o})}}=\left(\frac{2-p}{p}\right)^{1 / 2} \text {. }
$$

In turn, Eqn (33), used in Eqn (11), yields

$$
1+z=\left(\frac{2-p}{p}\right)^{1 / 2}
$$

By inspection of Eqns (35) \& (36) we see that they indeed imply Eqn (31). Once again, we have found that the Milne model exactly describes the dynamics of an empty universe.

Comparing Eqn (29) with Eqn (18) we see that Eqn (29) leads to an expression for the luminosity distance which underestimates the correct one by the factor of $t_{\mathrm{o}} / t_{\mathrm{e}}$. We have $t_{\mathrm{o}} / t_{\mathrm{e}}=\left(t_{\mathrm{o}} / \tau_{\mathrm{o}}\right) \cdot\left(\tau_{\mathrm{o}} / t_{\mathrm{e}}\right)=\gamma(1+z)=$ $\left[(1+z)^{2}+1\right] / 2$. Using Eqn (31) in Eqn (30) we obtain

$$
D^{(\mathrm{DL})}=(1+z)^{-1} \frac{c}{H_{\mathrm{o}}^{(\mathrm{o})}} \frac{(1+z)^{2}-1}{(1+z)^{2}+1},
$$

therefore

$$
D_{\mathrm{L}}^{(\mathrm{DL})}=(1+z) D^{(\mathrm{DL})}=\frac{c}{H_{\mathrm{o}}} \frac{(1+z)^{2}-1}{(1+z)^{2}+1} .
$$

Here, $H_{\mathrm{o}}$ denotes the value of the Hubble constant at the observing point at the time of observation and for simplicity we have omitted the superscript (o). Combined with Eqn (25), Eqn (38) yields the function shown in Figure 1 as a thin solid line. Davis \& Lineweaver did not provide an explicit formula for the magnitude-redshift relation in $\mathrm{SR}$, but this line closely follows their plotted curve.

The thick solid line in Figure 1 shows the magnitude-redshift relation for the currently favoured cosmological model: a flat universe with a nonzero 
cosmological constant, $\Omega_{\mathrm{m}}=0.28$ and $\Omega_{\Lambda}=0.72 .{ }^{1}$ Useful expressions for the luminosity distance in this model are given in Chodorowski (2005). We see that the thin solid line (prediction of SR according to Davis \& Lineweaver) is very distant from the thick solid line. Using the supernovae data of Perlmutter et al. (1999), Davis \& Lineweaver verified that the model given by the thin line is ruled out at more than $23 \sigma$ ' compared with the currently favoured model.

On the other hand, the dotted line (correct prediction of SR) follows the thick solid line much more closely. Given the data by Perlmutter et al. (1999), how close is the prediction of SR to that of the favoured model? The answer is provided by Perlmutter et al. themselves: Their analysis yielded the constraint $0.8 \Omega_{\mathrm{m}}-0.6 \Omega_{\Lambda}=-0.2 \pm 0.1$. An empty universe corresponds to $\Omega_{\mathrm{m}}=\Omega_{\Lambda}=0$, hence for SR, $0.8 \Omega_{\mathrm{m}}-0.6 \Omega_{\Lambda}=0$. We see thus that within two standard deviations, the data was consistent with an empty universe! This is not to defend this model as a viable alternative to the currently favoured model. From our mere existence we know the universe is not empty. A host of observational evidence consistently points towards the currently favoured model. This is only to say that a few years ago, the evidence from supernovae data alone for the accelerated expansion of the universe was not so strong, and the assumption of its purely kinematic expansion at low redshifts could then serve as a reasonable starting approximation.

Since 1999 the supernovae Ia data has improved. In particular, the analysis of Tonry et al. (2003) yielded $\Omega_{\Lambda}-1.4 \Omega_{\mathrm{m}}=0.35 \pm 0.14$. This constituted a modest improvement over the result of Perlmutter et al., implying the present acceleration of the universe's expansion to be detected at a $2.5 \sigma$ confidence. ${ }^{2}$ A significant improvement was achieved by discovering and observing supernovae Ia at $z>1$ with the Hubble Space Telescope. Figure 8 of Riess et al. (2004) shows the resulting joint confidence intervals for $\left(\Omega_{\mathrm{m}}, \Omega_{\Lambda}\right)$ from SNe Ia. In this figure, the point $\Omega_{\mathrm{m}}=\Omega_{\Lambda}=0$ lies outside shown confidence contours of $99.7 \%$. Riess et al. claim that 'with the current sample, the $4 \sigma$ confidence intervals (i.e., $>99.99 \%$ confidence) are now fully contained within the region where $\Omega_{\Lambda}>0$ '. Similar results of the analysis of the Riess et al. sample have been obtained independently by Wright (2005).

An empty universe is thus not a viable cosmological model, but remains a useful reference model when comparing predictions of various cosmological models. Figure 7 of Riess et al. shows the magnitude-redshift diagram for $\mathrm{SN}$ Ia in a residual form, relative to an empty universe model. Being eternally coasting, this model has

\footnotetext{
${ }^{1}$ These are recent estimates of the cosmological parameters $\Omega_{m}$ and $\Omega_{\Lambda}$ from the magnitude-redshift relation of observed high-redshift supernovae, combined with other observational constraints. See, for example, Tonry et al. (2003).

${ }^{2}$ From supernovae alone. Combined with the constraint of a flat universe, strongly supported by the CMB observations, the data of Tonry et al. yielded $\Omega_{\mathrm{m}}=0.28 \pm 0.05$ and $\Omega_{\Lambda}=0.72 \pm 0.05$, implying a currently accelerating universe at much higher confidence.
}

a vanishing deceleration parameter, so it naturally separates accelerating from decelerating models. Also, it is evident from figure 7 of Riess et al. that the model fits the data much better than an Einstein-de Sitter universe $\left(\Omega_{\mathrm{m}}=1, \Omega_{\Lambda}=0\right)$.

\section{Summary}

We have derived the angular diameter distance $D_{\mathrm{A}}$ and the luminosity distance $D_{\mathrm{L}}$ in the Milne kinematic cosmological model using only special relativistic concepts. In the derivations, the central role was played by the special relativistic Doppler formula for photons' redshift. We have found that $D_{\mathrm{L}}=(1+z)^{2} D_{\mathrm{A}}$, in accordance with general relativity. The derived formulae are identical to these corresponding to an empty universe in the FRW cosmology. We have shown where Davis \& Lineweaver failed to correctly derive the luminosity distance. Finally, we have presented the resulting magnitude-redshift diagram for supernovae Ia. While the prediction of special relativity according to Davis \& Lineweaver is far away from that for the currently favoured cosmological model, the correct prediction of special relativity follows the favoured model much more closely. Though not a viable alternative to the currently favoured model, the Milne model has great pedagogical value, elucidating the kinematic aspect of the universe's expansion. In the context of current research on supernovae Ia, it remains a useful reference model when comparing predictions of various cosmological models.

\section{Acknowledgments}

This research has been supported in part by the Polish State Committee for Scientific Research grant no. 1 P03D 012 26, allocated for the period 2004-2007.

\section{References}

Chodorowski, M. J. 2005, AmJPh, 73, 639

Davis, T. M., \& Lineweaver, C. H. 2004, PASA, 21, 97

Longair, M. S. 2003, Theoretical Concepts in Physics, 2nd edn (Cambridge: Cambridge University Press), 543

Milne, E. A. 1933, ZA, 6, 1

Peacock, J. A. 1999, Cosmological Physics (Cambridge: Cambridge University Press), 88

Perlmutter, S., et al. 1997, ApJ, 483, 565

Perlmutter, S., et al. 1999, ApJ, 517, 565

Perlmutter, S. 2003, PhT, 56, 53

Riess, A. G., et al. 2004, ApJ, 607, 665

Rindler, W. 1977, Essential Relativity, 2nd edn (New York: Springer), Sec. 9.4

Tonry, J. L., et al. 2003, ApJ, 594, 1

Wright, E. L. 2005, www.astro.ucla.edu/ $\sim$ wright/ sne_cosmology.html 